\title{
On ideal transforms of noetherian rings, I
}

\author{
By \\ Jun-ichi NisHimURA
}

\section{Introduction}

In [3], Matijevic has given a nice generalization of Krull-Akizuki Theorem:

Let $A$ be a noetherian ring. We define the total transform $T(A)$ of $A$ as follows: $T(A)=\{a \in Q(A)$ (=the total quotient ring of $A$ ); the conductor of $a$ to $A$ contains a power of a finite product of maximal ideals of $A$.

Theorem: Let $B$ be an $A$-algebra contained in $T(A)$. Then $B / x B$ is a finite $A$-module for each non-zero-divisor $x$ in $A$.

COROLlary: If $A$ is reduced, $B$ is always noetherian.

Proof consists of three steps:

Step 1. For any $b$ in $B$, there exists a $k>0$ such that $b \in A x^{-k}+x B$.

Step 2. The descending chain of ideals $I_{h}=\left(x^{h} B \cap A, x A\right)$ stabilizes for some $h=n$.

Step 3. $B$ is contained in $A x^{-n}+x B$. This gives the assertion.

In this article, we give a general theory on ideal transforms of noetherian rings using THEOREM and Corollary. We also apply the technique of ProOF to some problems on the integral closures of noetherian domains.

In section 1 , we begin by giving an elementary lemma on ideal transforms of noetherian rings. Then we treat the total transform of a local domain $(A, \mathfrak{n})$, which we call the global transform of $A$ and write by $A(\mathfrak{m})$. Proposition (1.2) gives a sufficient condition of $A(\mathfrak{m})$ to be integral over $A$. Proposition (1.3) shows that, for a henselian local domain $(A, \mathfrak{m})$ of dimension greater than one the derived normal ring (=the integral closure of $A$ in its field of quotients) $\bar{A}=\bigcap_{j=1}^{k} \bar{A}_{a_{j}}$, where $\mathfrak{m}=a_{1} A+\cdots+a_{k} A$. This replaces [6, Proposition 5], so that we can constantly use henselizations instead of completions in the proof of [6, Proposition 6]. Lemma (1.4) is easy, but $A(\mathfrak{m})$ being noetherian is essential. Theorem (1.6) gives the relation between $A(\mathfrak{m})$ and the integral closure $\tilde{A}$ of $A$ in $A(\mathfrak{m})$. Theorem (1.6) seems to be already known (cf. [3], [7], [8]), but we have not seen a complete proof. Corollary (1.7) gives a necessary (and sufficient) condition that $A(\mathfrak{m})$ is integral over $A$. Corollary (1.8) shows the explicit form of the ring which lies between $\tilde{A}$ and $A(\mathfrak{m})$. 
In section 2, we continue to investigate the global transform of a local ring, but not necessarily an integral domain. We begin with Proposition (2.1) in order that we can apply COROLLARY and its related results to non-reduced cases (cf. Proposition (2.2), Corollary (2.4), Theorem (2.6) and Proposition (2.7)). Proposition (2.3) gives criteria of $A(\mathfrak{m})$ to be finite over $A$ (or integral over $A$ ) in terms of associated primes of its completion $\hat{A}$. The proof of Proposition (2.3) is an easy exercise of commutative rings, but the union of Corollary (1.7) and Proposition (2.3) gives another proof of [5, (33.11)]:

Let $\vec{p}$ be a height one prime ideal of the derived normal ring of a noetherian domain $A$. Then $\mathfrak{p}=\bar{p} \cap A$ is an associated prime ideal of a principal ideal of $A$ (i. e. prof $A_{p}=1$ ).

In Theorem (2.6), we reduce the finiteness (or integralness) problem of a general ideal transform $A(I)$ to the same problem of global transforms of local rings $A_{\mathfrak{p}}$, where $\mathfrak{p}$ are prime ideals of $A$ containing $I$. Consequently, we get an elementary proof of Ferrand-Raynaud [1, Proposition 1.1].

In section 3 , we give criteria of the derived normal ring of a noetherian domain $A$ to be a finite $A$-module. Propositions (3.1) and (3.2) are slight generalizations of $[5,(35.3)]$ or $[4$, Lemma, p. 239]. We hope the hypotheses given here are more acceptable than that of $[5,(35.3)]$.

Finally, we emphasis that, even in Propositions (3.1) and (3.2), the technique of PROOF is essential.

\section{Notations and terminology}

In this paper, we mean by a ring a commutative ring with identity and by a local ring (resp. a semi-local ring) a noetherian ring with only one maximal ideal (resp. with finite number of maximal ideals). For a semi-local ring $\left(A, \mathfrak{m}_{1}\right.$, $\cdots, \mathfrak{m}_{r}$ ) with radical $\mathfrak{m}=\mathfrak{n t}_{1} \cdots \mathfrak{n}_{r}, \hat{A}$ denotes the completion of $A$ with respect to m. Let $I$ be an ideal of a ring $A$, we define the ideal transform $A(I)$ of $A$ with respect to $I$ as follows:

$$
\begin{gathered}
A(I)=\{a \in Q(A) \text { ( }=\text { the total quotient ring of } A) \text {; there exists } \\
\text { a natural number } \left.n \text { such that } I^{n} a \subset A\right\} .
\end{gathered}
$$

We assume $I$ contains a non-zero-divisor of $A$, whenever we define $A(I)$.

We call a semi-local domain $A$ a well-branched ring if the number of maximal ideals of $A$ is the same as that of $\bar{A}$ (=the derived normal ring of $A$ ). Let $A, B$ be semi-local domains with common field of quotients. We call $B$ a well-branched over-ring of $A$, if $B$ is a finite $A$-module and a well-branched ring.

Ass $(A)$ denotes the set of associated primes of $A$ and for a local ring $(A, \mathfrak{m})$ prof $A$ means the depth of $A$ with respect to $\mathfrak{m}$. 


\section{Global transforms of local domains}

Lemma (1.1) Let $I$ be an ideal of a noetherian ring $A$. Assume $I$ contains a non-zero-divisor of $A$. Hence we can write $I=a_{1} A+\cdots+a_{k} A$ with non-zerodivisor $a_{j}$ in $A$. Then

$$
A(I)=\bigcap_{j=1}^{k} A_{a_{j}} .
$$

Therefore, if $B$ is a flat A-algebra, we have:

(1.1.2) $\quad B(I B)=A(I) \otimes_{A} B$.

In particular, let $\left(A, \mathfrak{m}_{1}, \cdots, \mathfrak{m}_{r}\right)$ be a semi-local ring with radical $\mathfrak{m}=\mathfrak{m}_{1} \cdots \mathfrak{m}_{r}$ (which contains a non-zero-divisor). For each maximal ideal $\mathfrak{m}_{i}$ :

(1.1.3) $\quad A(\mathfrak{m})_{m_{i}}=A_{m_{i}}\left(\mathfrak{m}_{i}\right)$.

For non-maximal prime ideal $\mathfrak{p}$ :

(1.1.4) $\quad A(\mathfrak{m})_{p}=A_{p}$.

Hence there exists a unique prime ideal a of $A(\mathfrak{m})$ such that:

(1.1.5) $\mathfrak{p}=\mathfrak{q} \cap A$ and $A(\mathfrak{m})_{\mathfrak{q}}=A_{\mathfrak{p}}$.

Proposition (1.2) (cf. [7, Remarque $]$ ) Let $(A, \mathfrak{m})$ be a local domain. Suppose $\operatorname{dim} \bar{A}_{\overline{i n}}>1$ for any maximal ideal $\overline{\mathfrak{m}}$ of $\bar{A}$ (=the derived normal ring of $A$ ). Then

(1.2.1) $\quad A(\mathfrak{m})$ is integral over $A$.

Hence

(1.2.2) (A(m), $\left.\mathfrak{n}_{1}, \cdots, \mathfrak{n}_{s}\right)$ is a semi-local domain.

(1.2.3) $\quad$ prof $A\left(\mathfrak{m}, \mathfrak{n}_{i}>1\right.$ for each maximal ideal $\mathfrak{n}_{i}$ of $A(\mathfrak{m})$.

Proof. Let $B=A(\mathfrak{m}) \cap \bar{A}$. Then $\left(B, \mathfrak{n}_{1}, \cdots, \mathfrak{n}_{s}\right)$ is a semi-local domain with radical $\mathfrak{n}=\mathfrak{n}_{1} \cdots \mathfrak{n}_{s}$ by COROLLARY.

We claim : $B(\mathfrak{n})=B$ and $A(\mathfrak{m})=B$.

1) $\mathfrak{n}: \mathfrak{n}=B$. Since $\mathfrak{n}: \mathfrak{n} \subset A(\mathfrak{m}): \mathfrak{m}=A(\mathfrak{m})$ and $\mathfrak{n}: \mathfrak{n} \subset \bar{B}=\bar{A}, \quad B \subset \mathfrak{n}: \mathfrak{n} \subset$ $A(\mathfrak{n}) \cap \bar{A}=B$.

2) $B(\mathfrak{n})=B . \quad$ As $\operatorname{dim} P_{\mathbf{n}_{i}}>1$ for any $i,\left(B: \mathfrak{n}_{i}\right) \mathfrak{n}_{i}=\mathfrak{n}_{i}$. Hence $B: \mathfrak{n}=\mathfrak{n}: \mathfrak{n}=B$.

3) $A(\mathfrak{m})=B$. Since $B$ is noetherian and $\operatorname{rad}(\mathfrak{m} B)=\mathfrak{n}, A(\mathfrak{m})=\bigcup_{\nu>0}\left(A: \mathfrak{m}^{2}\right) \subset$ $\bigcup_{\nu>0}\left(B: \mathfrak{m}^{\nu}\right)=\bigcup_{\mu>0}\left(B: \mathfrak{n}^{\prime}\right)=B(\mathfrak{n})=B$.

Proposition (1.3) Let $(A, \mathfrak{m})$ be a henselian local domain of dimension greater than one. Write $\mathfrak{m}=a_{1} A+\cdots+a_{k} A$. Then $\bar{A}=\bigcap_{j=1}^{k} \bar{A}_{a_{i}}$. 
Proof is easy, because $A(\mathfrak{m})=\bigcap_{j=1}^{k} A_{a_{j}}$ is integral over $A$ by (1.2.1).

Lemma (1.4) Let $\left(A, \mathfrak{m}_{1}, \cdots, \mathfrak{m}_{r}\right),\left(B, \mathfrak{n}_{1}, \cdots, \mathfrak{n}_{s}\right)$ be semi-local domains with common field of quotients. If $B$ is a finite $A$-module, than $B(\mathfrak{n})$ is a finite $A(\mathfrak{m})$-module.

Proposition (1.5) Let $(A, \mathfrak{m})$ be a local domain of dimension greater than one. Then

(1.5.1) $\left(A(\mathfrak{m}), \mathfrak{n}_{1}, \cdots, \mathfrak{n}_{s}\right)$ is a semi-local domain with $\operatorname{dim} A(\mathfrak{m})=\operatorname{dim} A$.

For each maximal ideal $\mathfrak{n}_{i}$ of $A(\mathfrak{m})$,

(1.5.2) $\quad \mathfrak{n}_{i} \cap A=\mathfrak{m}$, and

(1.5.3) $\quad$ prof $A(\mathfrak{m})_{\mathfrak{n}_{i}}>1$.

Proof. Let $\left(B, \mathfrak{m}_{1}^{\prime}, \cdots, \mathfrak{m}_{r}^{\prime}\right)$ be a well-branched over-ring of $A$ with radical $\mathfrak{m}^{\prime}=\mathfrak{m}_{1}^{\prime} \cdots \mathfrak{m}_{r}^{\prime}$. Suppose $\operatorname{dim} B_{\mathfrak{m}_{i}^{\prime}}>1$ iff $i \leqq r_{0}(\leqq r)$. Then $B\left(\mathfrak{m}^{\prime}\right)=B_{S}\left(\mathfrak{m}^{\prime}\right)$, where $S=B-\bigcup_{i \leq r_{0}} \mathfrak{m}_{i}^{\prime}$ by (1.1.3). Since $B_{S}\left(\mathfrak{m}^{\prime}{ }_{s}\right)$ is integral over $B_{S}$ and $B\left(\mathfrak{m}^{\prime}\right)$ is a finite $A(\mathfrak{m})$-module by $(1.2 .1)$ and Lemma $(1.4),\left(A(\mathfrak{m}), \mathfrak{n}_{1}, \cdots, \mathfrak{n}_{s}\right)$ is a semi-local domain with $\mathfrak{n}_{i} \cap A=\mathfrak{m}$ and $\operatorname{rad}(\mathfrak{m} A(\mathfrak{m}))=\mathfrak{n}_{1} \cdots \mathfrak{n}_{s}$. Therefore we get the assertions.

Theorem (1. 6) (cf. [7, Lemme 3], [8, Corollaires ( I 4) et ( I 10)]) Let (A, m) be a local domain with field of quotients $K$. Let $P=\left\{\bar{p}_{1}, \cdots, \bar{p}_{t}\right\}$ be the set of height one maximal ideals of $\bar{A}$ (which may be empty).

Put $\tilde{A}=A(\mathfrak{m}) \cap \bar{A}$ (=the integral closure of $A$ in $A(\mathfrak{m}))$. Then

(1.6.1) $\left(\tilde{A}, \tilde{\mathfrak{n}}_{1}, \cdots, \tilde{\mathfrak{n}}_{r}\right)$ is a semi-local domain with radical $\tilde{\mathfrak{n}}=\tilde{\mathfrak{m}}_{1} \cdots \tilde{\mathfrak{m}}_{r}$.

(1.6.2) $\tilde{A}=A(\mathfrak{m}) \cap \bar{A}_{\bar{p}_{1}} \cap \cdots \cap \bar{A}_{\bar{p}_{t}}$.

(1.6.3) $\bar{A}=\overline{A(\mathfrak{m t})} \cap \bar{A}_{\bar{p}_{1}} \cap \cdots \cap \bar{A}_{\bar{p}_{t}}$.

(1.6.4) $\quad \tilde{A}(\mathfrak{i} \mathrm{i})=A(\mathfrak{n t})$.

Divide the set $M=\left\{\tilde{\mathfrak{m}}_{1}, \cdots, \tilde{\mathfrak{n}}_{r}\right\}$ of maximal ideals of $\tilde{A}$ into three classes:

$$
\begin{array}{ll}
M_{0}=\left\{\tilde{u}_{k} \in M ; \operatorname{dim} \tilde{A}_{\tilde{\mathfrak{m}}_{k}}=0\right\}, & M_{1}=\left\{\tilde{\mathrm{m}}_{j} \in M ; \operatorname{dim} \tilde{A}_{\tilde{\mathfrak{m}}_{j}}=1\right\} \quad \text { and } \\
M_{2}=\left\{\tilde{\mathrm{u}}_{i} \in M ; \operatorname{dim} \tilde{A}_{\tilde{\mathrm{m}}_{i}}>1\right\} . & \text { Then }
\end{array}
$$

(1.6.5) $A(\mathfrak{m})=\tilde{A}_{S}$, where $S=\tilde{A}-{\widetilde{\tilde{m}_{i} \in M_{2}}}_{\mathfrak{m}_{i}} \quad$ if $M_{2}$ is empty, $\left.S=A-\{0\}\right)$.

$P$ is not empty if and only if $M_{1}$ is not empty and:

$$
\bar{A}_{\overline{\mathfrak{p}}_{1}} \cap \cdots \cap \bar{A}_{\overline{\mathfrak{p}}_{t}}=\tilde{A}_{T} \text {, where } T=\tilde{A}-\underset{\tilde{m}_{j} \in M_{1}}{\bigcup} \tilde{\mathfrak{m}}_{j} .
$$

Proof. 1) Let $\left(B, \mathfrak{n}_{1}, \cdots, \mathfrak{n}_{s+t}\right)$ be a well-branched over-ring of $A$ such that $\operatorname{dim} B_{\mathfrak{n}_{i}}>1$ iff $i \leqq s$ ( $s$ may be zero). 
Then

$$
\begin{aligned}
A & =A(\mathfrak{m}) \cap \bar{A}=A(\mathfrak{m}) \cap B(\mathfrak{n}) \cap \bar{B}=A(\mathfrak{m}) \cap\left(\bigcap_{i=1}^{s} B_{\mathbf{z}_{i}}\left(\mathfrak{n}_{i}\right)\right) \cap\left(\bigcap_{j=1}^{s+t} B_{\mathfrak{n}:}\right) \\
& =A(\mathfrak{m}) \cap B(\mathfrak{n}) \cap\left(\bigcap_{j=s+1}^{s+t} \bar{B}_{\mathfrak{n}_{j}}\right)=A(\mathfrak{m}) \cap \bar{A}_{\overline{\mathfrak{p}}_{1}} \cap \cdots \cap \bar{A}_{\overline{\mathfrak{p}}_{t}} .
\end{aligned}
$$

$\bar{A}_{\bar{p}_{j}}=\bar{B}_{\mathfrak{n}_{s+j}}$ are discrete valuation rings (cf. $[5,(33.2)]$ ).

2) On the other hand,

$$
\begin{aligned}
\bar{A}=\bar{B}= & \bigcap_{i=1}^{s+t} \bar{B}_{\mathfrak{n}_{i}}=\left(\bigcap_{i=1}^{s} \bar{B}_{\mathfrak{n}_{i}}\right) \cap\left(\bigcap_{j=s+1}^{s+t} \bar{B}_{\mathfrak{n}_{j}}\right) \\
= & \overline{B(\mathfrak{n})} \cap\left(\bigcap_{j=1}^{t} \bar{A}_{\bar{p}_{j}}\right)=\overline{A(\mathfrak{m})} \cap \bar{A}_{\mathfrak{p}_{1}} \cap \cdots \cap \bar{A}_{\bar{p}_{t}} \\
& \quad \text { (cf. (1.2.1), Lemma (1.4)). }
\end{aligned}
$$

3) Since $A(\mathfrak{m})$ is noetherian, $A(\mathfrak{n t}) \subset \tilde{A}(\tilde{\mathfrak{n}}) \subset A(\mathfrak{m})((\mathfrak{n i}) \subset A(\mathfrak{m})(\mathfrak{m i t})=A(\mathfrak{m})$.

4) Consider the pair of semi-local domains $\tilde{A}_{S}$ and $A(\mathfrak{m})$ with common field of quotients. Since $A(\mathfrak{m})_{S}=\tilde{A}(\tilde{\mathfrak{m}})_{S}=\tilde{A}(\tilde{\mathfrak{m}})=A(\mathfrak{m}), \tilde{A}_{S}$ is integrally closed in $A(\mathfrak{m})$. As $\tilde{A}(\tilde{\mathfrak{m}})_{\tilde{\mathfrak{m}}_{i}}=\tilde{A}_{\tilde{\mathfrak{m}}_{i}}\left(\tilde{\mathfrak{m}}_{i}\right)=A(\mathfrak{m})_{\tilde{\mathfrak{m}}_{i}}$ for each $\tilde{\mathfrak{m}}_{i}$ in $M_{2}$, for any prime ideal $\tilde{\mathfrak{p}}$ of $\tilde{A}_{S}$, there always exists a prime ideal $\mathfrak{q}$ of $A(\mathfrak{m})$ such that $\mathfrak{q} \cap \tilde{A}_{S}=\tilde{\mathfrak{p}}$ by (1.5.2) and (1.1.4). Hence $\tilde{A}_{S}=A(\mathfrak{m})$ by $[5,(33.1)]$.

5) Since $\bar{A}_{S}=\tilde{\bar{A}}_{S}=\overline{A(\mathfrak{m})}=\overline{B(\mathfrak{n})}$ has no maximal ideal of height one,

$$
\begin{aligned}
& \tilde{A}_{T}=A(\mathfrak{m})_{T} \cap\left(\bar{A}_{\bar{p}_{1}}\right)_{T} \cap \cdots \cap\left(\bar{A}_{\bar{p}_{t}}\right)_{T}=\tilde{A}(\tilde{\mathfrak{m}})_{T} \cap \bigcap_{j=1}^{t} \bar{A}_{\bar{p}_{j}} \\
&=K \cap \bar{A}_{\bar{p}_{1}} \cap \cdots \cap \bar{A}_{\mathfrak{p}_{t}}=\bar{A}_{\overline{\mathfrak{p}}_{1}} \cap \cdots \cap \bar{A}_{\overline{\mathfrak{p}}_{t} .} .
\end{aligned}
$$

Corollary (1.7) Let $(A, \mathfrak{m})$ be a local domain. Then $A(\mathfrak{n})$ is integral over $A$ if and only if the derived normal ring $\bar{A}$ of $A$ has no maximal ideal of height one (cf. (1.2.1)).

Corollary (1.8) With the notations as in Theorem (1.6). Let $C$ be a ring such that $\tilde{A} \subset C \subset A(\mathfrak{m})$, then $C=A(\mathfrak{m}) \cap\left(\widehat{\widetilde{p}} \in Q Q_{\mathfrak{p}_{j}}\right)$, where $Q$ is a subset of $P$.

Department of Mathematics, Kyoto University

\section{References}

[1] D. Ferrand and M. Raynaud: Fibres formelles d'un anneau local noethérien, Ann. Sci. Ecole Norm. Sup, (4) 3 (1970), 295-311.

[2] H. Kurke, G. Pfister und M. Roczen: Henselsche Ringe und algebraische Geometrie, VEB Deutscher Verlag der Wissenschaften, 1975.

[3] J. Matijevic: Maximal ideal transforms of noetherian rings, Proc. Amer. Math. Soc., 54 (1976), 49-52. 
[4] H. Matsumura: Commutative algebra, Benjamin, 1970.

[5] M. Nagata: Local rings, Interscience, 1962.

[6] J. Nishimura: Note on integral closures of a noetherian integral domain, Jour. Math. Kyoto Univ., 16 (1976), 117-122.

[7] J. Querré: Sur un théorème de Mori-Nagata, C. R. Acad. Sc. Paris, t. 285 (1977), 323-324.

[8] H. Seydi: Sur la transformation de Nagata (preprint). 